\title{
Optimization of CMS HCAL Parameters Using Hanging File Test Data
}

\author{
D. Green \\ Fermi National Accelerator Laboratory \\ P.O. Box 500, Batavia, Illinois 60510
}

June 1994 


\section{Disclaimer}

This report was prepared as an account of work sponsored by an agency of the United States Government. Neither the United States Government nor any agency thereof, nor any of their employees, makes any warranty, express or implied, or assumes any legal liability or responsibility for the accuracy, completeness, or usefulness of any information, apparatus, product, or process disclosed, or represents that its use would not infringe privately owned rights. Reference herein to any specific commercial product, process, or service by trade name, trademark, manufacturer, or otherwise, does not necessarily constitute or imply its endorsement, recommendation, or favoring by the United States Government or any agency thereof. The views and opinions of authors expressed herein do not necessarily state or reflect those of the United States Government or any agency thereof. 


\title{
OPTIMIZATION OF CMS HCAL
}

PARAMETERS USING HANGING

FILE TEST DATA

\author{
Dan Green \\ Fermilab
}

\section{Introduction}

There are 2 basic choices for the magnetic solenoid coil location in large collider experiments. One can place the coil inside the calorimeter radius as in CDF, D0, ATLAS, and SDC. Alternatively, one can place the coil outside the calorimetry as in SLD, ZEUS, and CMS. There are clearly definite advantages to both schemes since different collaborations have chosen different topologies.

In this note we examine the consequences of the second choice, which places no significant material in front of the EM compartment, but severely limits the total depth of hadronic calorimetry (HCAL) available before the appearance of inert material (the coil).

\section{Data Sets and Model}

Several data sets are useful in performing this study. First there is data with 50,200 , and $450 \mathrm{GeV}$ hadrons incident on a very deep neutrino detector [1]. The total depth is 19.2 absorption lengths. Each sample is read out separately and independently. The unit, absorption lengths, will be implicit in all the discussions of depth which follow. The detector has 0.7 sampling, which is a bit coarse for some purposes, but it has the maximum energy available and the maximum depth.

Using this data a model of the CMS detector was made. The coil, which is $\mathrm{Al}$, was assumed to be modeled as completely inert Fe of depth $=2$ samples, or 1.4. Clearly, completely dropping the readout layers is a worst case estimate, as EM energy in the coil will leak out more from Al than Fe. The energy in the first HCAL compartment was called e1, followed by a distinct "exit weight" readout of depth 0.7 just upstream of the coil, called e2. The coil Fe is immediately followed by an active "massless gap" readout called e3. The last element is a "tailcatcher" of Fe absorber and readout, called e4.

In a typical run e1 was 4.5 deep, with the exit weight at depth 5.2. The coil was 1.4 deep, so that the massless gap occurred at depth 6.6 , followed by the tailcatcher extending to depth 8.7.

A similar model was constructed using the "hanging file" data set [2]. The highest energy hadron data, $250 \mathrm{GeV}$ was used. The sampling was 40 layers of $1 / 8^{\prime \prime} \mathrm{Pb}(\mathrm{EM})$ followed by 55 layers of 1 " Fe (HCAL), where each layer had an independent readout. This data was of greatest use in studying the sampling fraction. In CMS there is a tradeoff between energy leakage, arguing for coarse sampling (and thus larger total depth), and stochastic error, arguing for fine 
sampling. In a typical run e1 was 4.5 deep, e2 was at 5.3 depth, the coil was 1.36 deep with e3 at 6.66 depth, and the tailcatcher was at 8.78 maximum depth. The full hanging file stack extended to a depth of 9.4 , compared to 19.2 for the neutrino detector data.

The fractional energy resolution for the fully active, full depth data set was $4.4 \%$ for the $450 \mathrm{GeV}$ neutrino data, and $5.3 \%$ for the $250 \mathrm{GeV}$ hanging file data. These resolutions are the benchmarks for "good" operation of the calorimeter.

\section{Optimizing Depth Segments}

The first studies were attempts to optimize the depth of the various HCAL depth segments. For this purpose, for each configuration the data was fit to a function of 3 parameters such as to minimize the rms error with respect to the fully active and deep measurements.

$$
E=e 1+W_{T E}^{*} e 2+W T M^{*} e 3+W T T^{*} e 4
$$

The "weights" are simply constants which do not depend on the shower development on an event by event basis.

The results obtained for the $450 \mathrm{GeV}$ neutrino data set are shown in Fig.1. To set the scale, for 5.2 depth upstream of the coil and a 2.1 deep tailcatcher, $83 \%$ of the energy appears in e1+e2, $8.0 \%$ is lost in the coil, and $9.2 \%$ is found in the tail on average.

In Fig.1a is shown the result for a variable total depth of e1 plus e2 upstream of the coil, with the tailcatcher thickness fixed at 2.1. Clearly, there is a steep reduction in $\mathrm{dE} / \mathrm{E}$ until an upstream depth of $\sim 6$ is reached. In Fig.1b is shown the result for a fixed upstream depth of e1 of 4.5 followed by an exit depth of 0.7 , or 5.2 total upstream depth. The tailcatcher depth is varied. Clearly, there is a steep decline in $\mathrm{dE} / \mathrm{E}$ until a depth of $\sim 2.5$ is achieved. For reference, the $4.4 \%$ unperturbed result is also shown on the graphs. With depth of 5.2 upstream and 2.1 of tailcatcher, the rms resolution is $5.8 \%$, or a $32 \%$ degradation.

The analogous result for the $250 \mathrm{GeV}$ hanging file data is shown in Fig.2. In this case, the tailcatcher depth is insensitive, since the lower energy showers deposit little energy beyond the coil. In fact, $96 \%$ of the total energy, on average, appears in a 5.2 depth upstream of the coil. Only $2 \%$ of the energy is lost in the coil, and $2.2 \%$ appears in the tailcatcher.

There is a steep fall of $\mathrm{dE} / \mathrm{E}$ with upstream depth, approaching the 5.3\% full depth resolution at $\sim 5.5 \mathrm{e} 1$ plus e2 upstream depth. At a depth of 5.2 upstream of the coil, the rms fractional resolution is $6.1 \%$, which is a $15 \%$ degradation with respect to the unperturbed, full depth, hanging file data. 


\section{Optimizing Sampling}

The fine sampling data available in the "hanging file" set was used to study the question of optimal sampling. A sampling of 2" and 4" was simulated by dropping the interleaved active layers. The depths were fixed at 5.2 upstream of the coil, and 2.1 downstream of the 1.4 deep coil. Within the 5.2 thickness, the sampling began with 1 " frequency, and at some boundary ended with 2" or 4" frequency. In Fig. 3 is shown the result of this study. Clearly, 4" sampling is too coarse. The benchmark is the $6.1 \%$ result shown in Fig.2.

Clearly, 2" sampling can be used after a depth of about 2.5 ( 0.74 is the EM $\mathrm{Pb}$ compartment in the hanging file data). Therefore, the choice of sampling is taken to be EM plus $~ 1.75$ with 1 " sampling followed by 2.7 (e1 plus e2) with 2 " Fe sampling. The final rms resolution, $\mathrm{dE} / \mathrm{E}$, is $6.7 \%$ which represents a $26 \%$ degradation of the "ideal" calorimeter which has $5.3 \% \mathrm{rms} \mathrm{dE} / \mathrm{E}$. Clearly, if much more energetic hadrons were incident on the calorimeter, the $1 " / 2$ " boundary location would have to retreat deeper into the calorimeter, albeit only logarithmically.

\section{Tail Reduction in the Energy Measurement}

The HCAL will be used to measure jets and to infer the presence of neutrinos by the existence of missing energy. For jet spectroscopy, other errors make the energy measurement not critical in determining the jet properties [3]. For the resolutions achieved in these studies, other errors will dominate in jet-jet mass, for example.

What is crucial is that the calorimeter not have tails, corresponding to catastrophic energy mismeasurement. These tails would swamp the expected signals due to supersymmetric particle production, for example. A concerted effort has been made to insure that the CMS optimization does not generate tails which would compromise the Physics at the LHC.

Shown in Fig.4 is the energy with respect to the incident beam energy for the "ideal" hanging file stack and the optimized stack. One pathological event has been removed so as to achieve the $6.7 \%$ resolution quoted in the previous section. Notice that with $\sim 200$ events in the plot there are no excursions beyond 20\% from the nominal beam momentum.

The pathological event is shown, in depth profile, in Fig.5. If Eq.1 is used to reconstruct the energy an error of $\sim 50 \%$ is made. Clearly, this event has put very little energy into the e1 and e2 compartments, has dropped most of the energy into the coil, and yet has died out before the massless gap and tailcatcher can sample the shower. In fact, there is $21 \%$ of the energy in e1 plus e2, and only $6.6 \%$ in e3 plus e4.

In an attempt to recover this event, and other pathological types, an extended fit with event by event weighting of the distinct depth readouts e1, e2, e3, and e4 was studied. The functional form which was tried was: 


$$
E=e 1+[a 1+b 1(e 2 / e 1)] e 2+[a 2+b 2(e 3 / e 1)] e 3+[a 3+b 3(e 4 / e 1)] e 4
$$

Note that for the average event, e2/e1 is 0.05 , while for the bad event, e2/e1 is 0.95 . Obviously, utilizing the event by event fluctuations in the shower development is the key to the repair of the measurement. In what follows, the results will be referred to as the 6 parameter fit in contrast to the 3 parameter fit using Eq.1.

The fractional energy resolution, with respect to the full calorimeter measurement, for all events is shown in Fig.6. Note that the 6 parameter fit has allowed the recovery of the $\sim 50 \%$ mismeasured event. There are no mismeasures more than $20 \%$ in this data set with the 6 parameter fit. There are only 2 events $(1 \%)$ beyond $10 \%$ deviation. The rms has been reduced to $6.8 \%$ compared to the $7.5 \%$ with the inclusion of the bad event in the full data set fit to 3 parameters. It appears that the tails of the energy distribution have been pulled in substantially.

The analogous results for the $450 \mathrm{GeV}$ neutrino data set are shown in Fig.7. There are no deviations beyond $20 \%$. However, it is clear that there is still some residual leakage effect. The rms fractional error is $5.7 \%$, reduced only slightly from the $5.8 \%$ achieved in the 3 parameter fit. However, in examining the 50 and $200 \mathrm{GeV}$ data sets it is clear that the 6 parameter fit is, in general, much superior in reducing the tails of the energy distributions.

\section{Tailcatcher Boundary}

In a "barrel" geometry the depth scales as $1 / \sin (\theta)$ where $\theta$ is the polar angle. At fixed $\mathrm{Pt}$, which defines most Physics processes, the energy also increases as $1 / \sin (\theta)$. Since the shower depth only increases logarithmically with the incident energy, there will come an angle below which the tailcatcher is not needed.

In order to get a first look at the location of this transition point the neutrino test data were used. The tailcatcher energy sample was ignored, and a 4 parameter fit was used which only employed the first terms in Eq.2 while dropping the 2 terms containing the energy e4. The massless gap (e3) was retained, however. The reconstructed $\mathrm{rms}$ fractional energy with this 4 parameter fit is shown in Fig.8. In Fig.8a the $450 \mathrm{GeV}$ hadron data is shown. Clearly, after $\sim 7.5$ upstream depth, no further large improvement occurs. Reconstructed energy distributions for 50,200 , and $450 \mathrm{GeV}$ incident beams are shown in Fig.8b. Clearly, for energies $450 \mathrm{GeV}$ and below, a depth greater than $\sim 7.5$ is sufficient to measure the energy well. The energy dependence of the minimum required depth is weak as expected. 


\section{Summary}

Existing test beam data sets were used to perform a preliminary optimization of the parameters of the CMS HCAL. It was found that, within the constraints of CMS, severe penalties on energy resolution, both the width and the tails, were not incurred. The price to be paid is in the readout, event by event, of several independent longitudinal segments. Having paid this price, the basic Physics appears not to be compromised.

\section{References}

1. A. Beretvas, private communication

2. A. Beretvas et. al., Nuc. Inst. and Meth. A329 50 (1993)

3. SDC Technical Design Report, April 1, 1992, SDC-92-201

4. D. Green, Fermilab TM-1883, April, 1994 

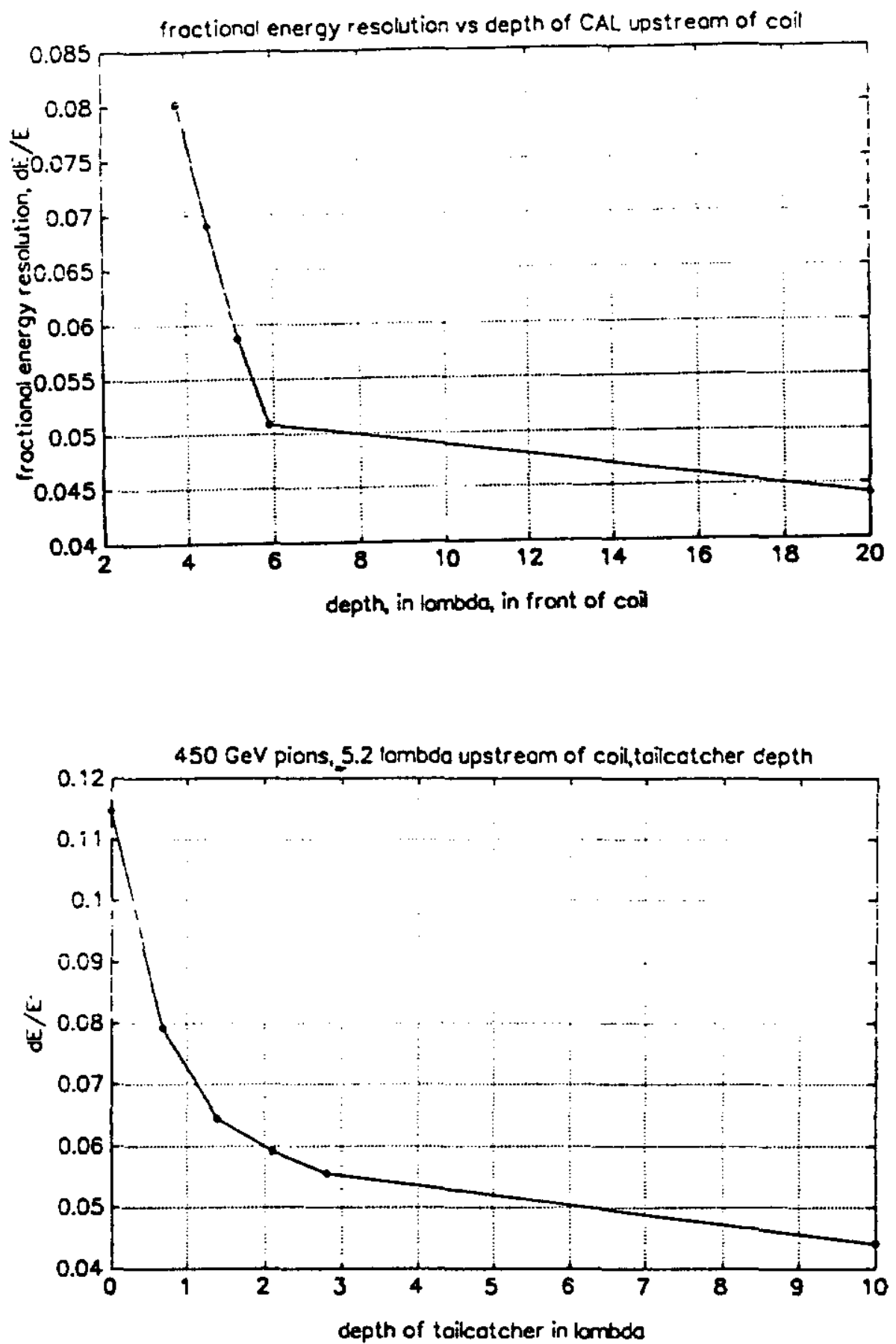

Figure 1. Data from neutrino detector with $450 \mathrm{GeV}$ pion test beam incident. Full detector depth is 19.2 absorption lengths

a. With tailcatcher depth fixed at 2.1, and coil fixed at 1.4, fractional energy resolution obtained in a 3 parameter fit as a function of depth upstream of the coil.

b. With upstream depth fixed at 5.2, and coil fixed at 1.4, fractional energy resolution obtained in a 3 parameter fit as a function of the depth of the tailcatcher. 


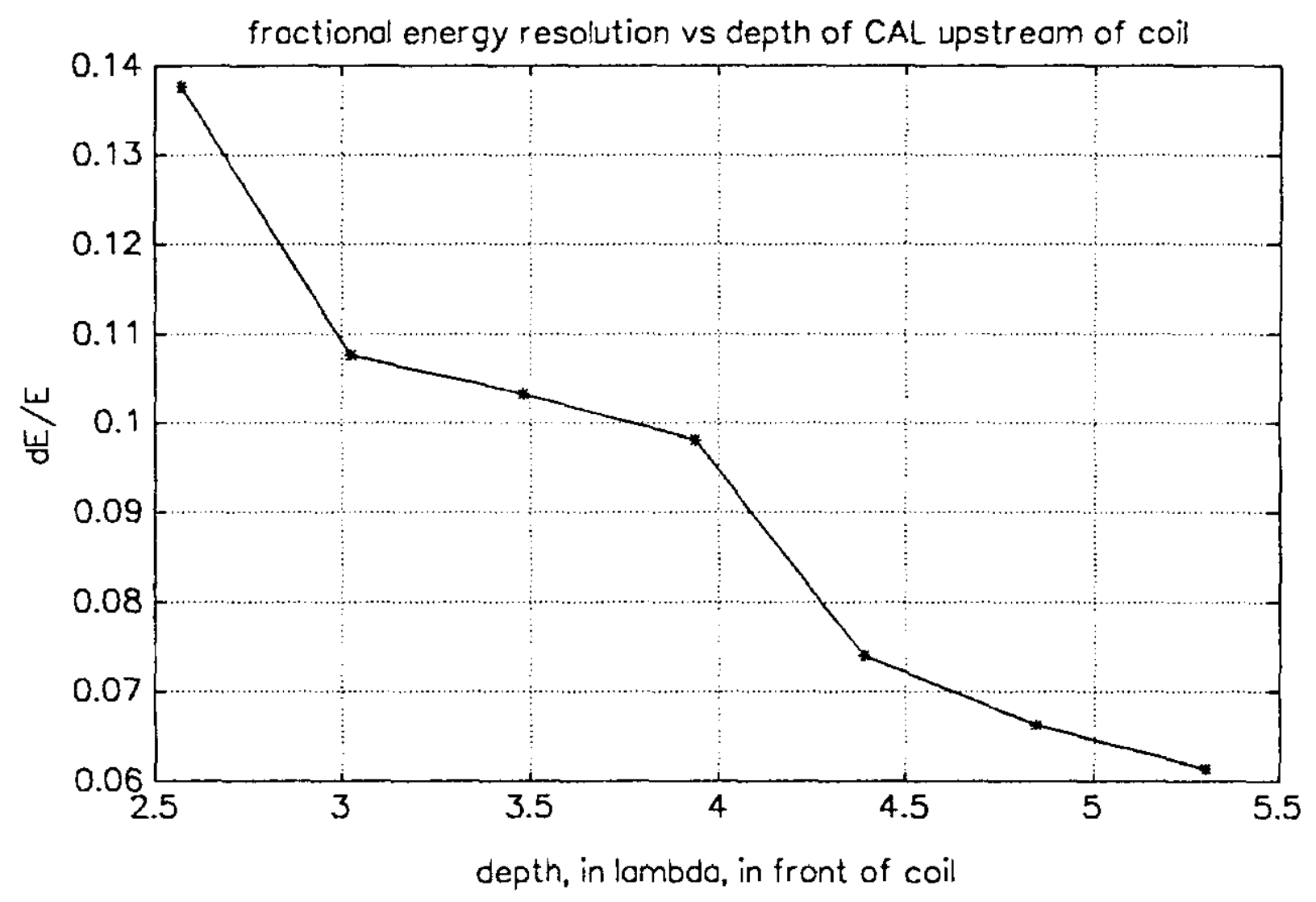




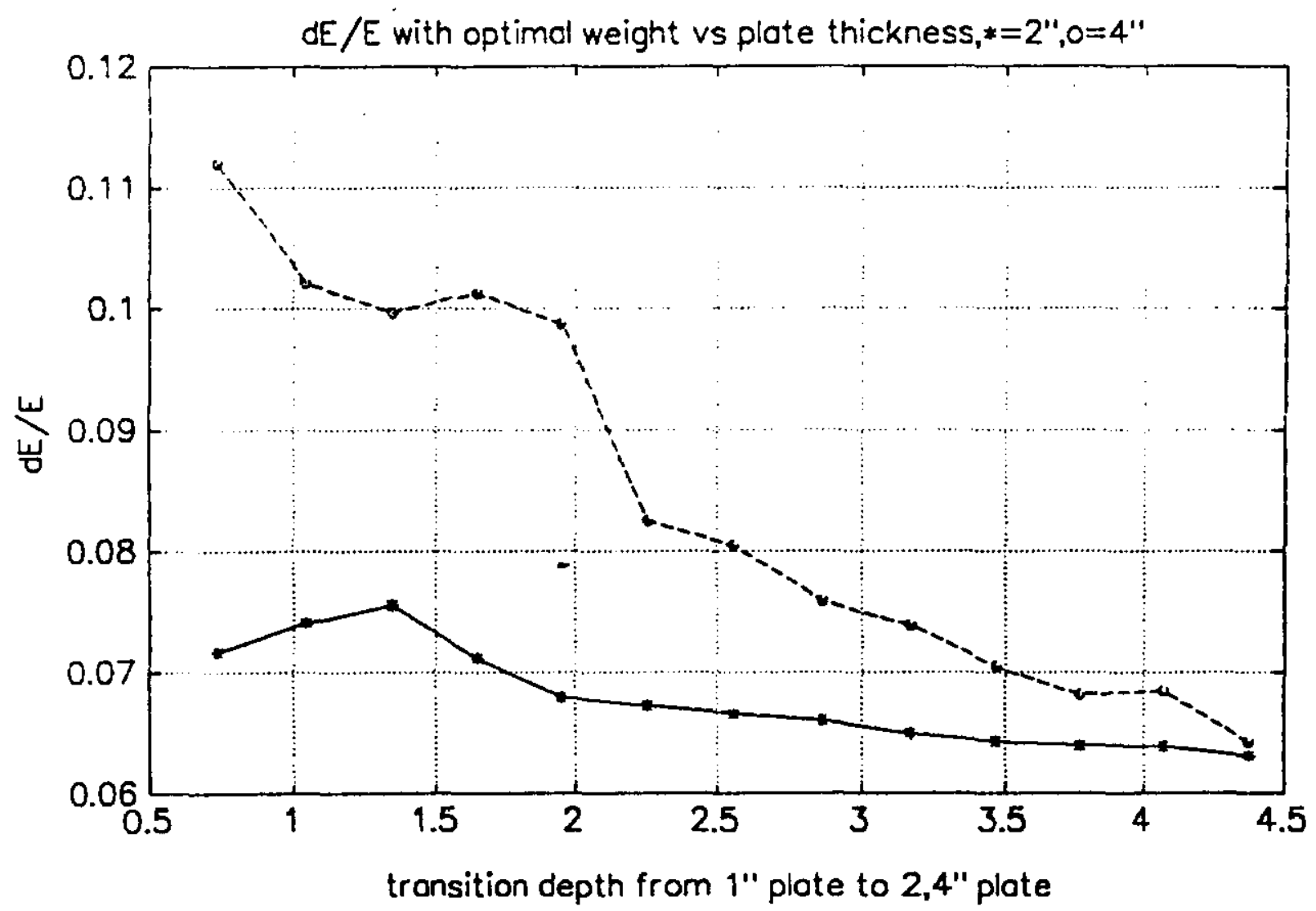

Figure 3. Data from the "hanging file" with $250 \mathrm{GeV}$ incident pions. The data was taken with 1" Fe plate sampling. With depth upstream of the coil fixed at 5.2, coil depth fixed at 1.4, and tailcatcher fixed at 2.1 a 3 parameter fit is made. The sampling is simulated to be coarser by a factor 2 and 4 by dropping interleaved readout layers. The EM compartment is 0.74 deep. The hadronic compartment is coarsened beyond a variable depth, and the resulting fractional energy resolution is plotted. 


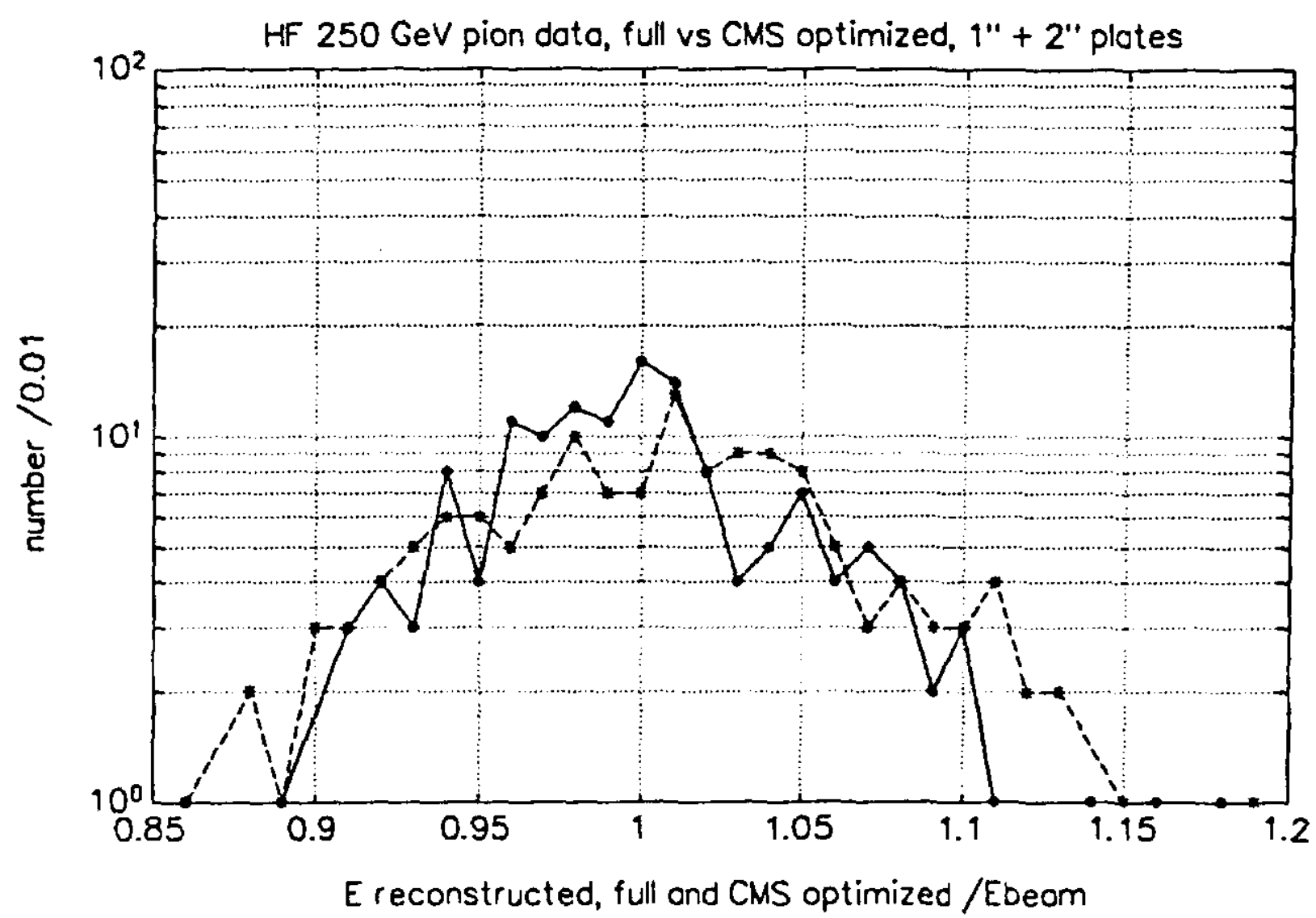

Figure 4. Data from the "hanging file" with $250 \mathrm{GeV}$ incident pions. The energy for the 1" Fe sampling with 9.4 depth and no inert material is plotted as the - - points/line. For comparison, the results of a 3 parameter fit with 5.2 upstream of a 1.4 coil followed by a 2.1 tailcatcher are shown as the *-.- points/line. The latter sampling consists of 12 plates of 1 " Fe followed by simulated 2" sampling. One badly fitted event is excluded from the plot. 


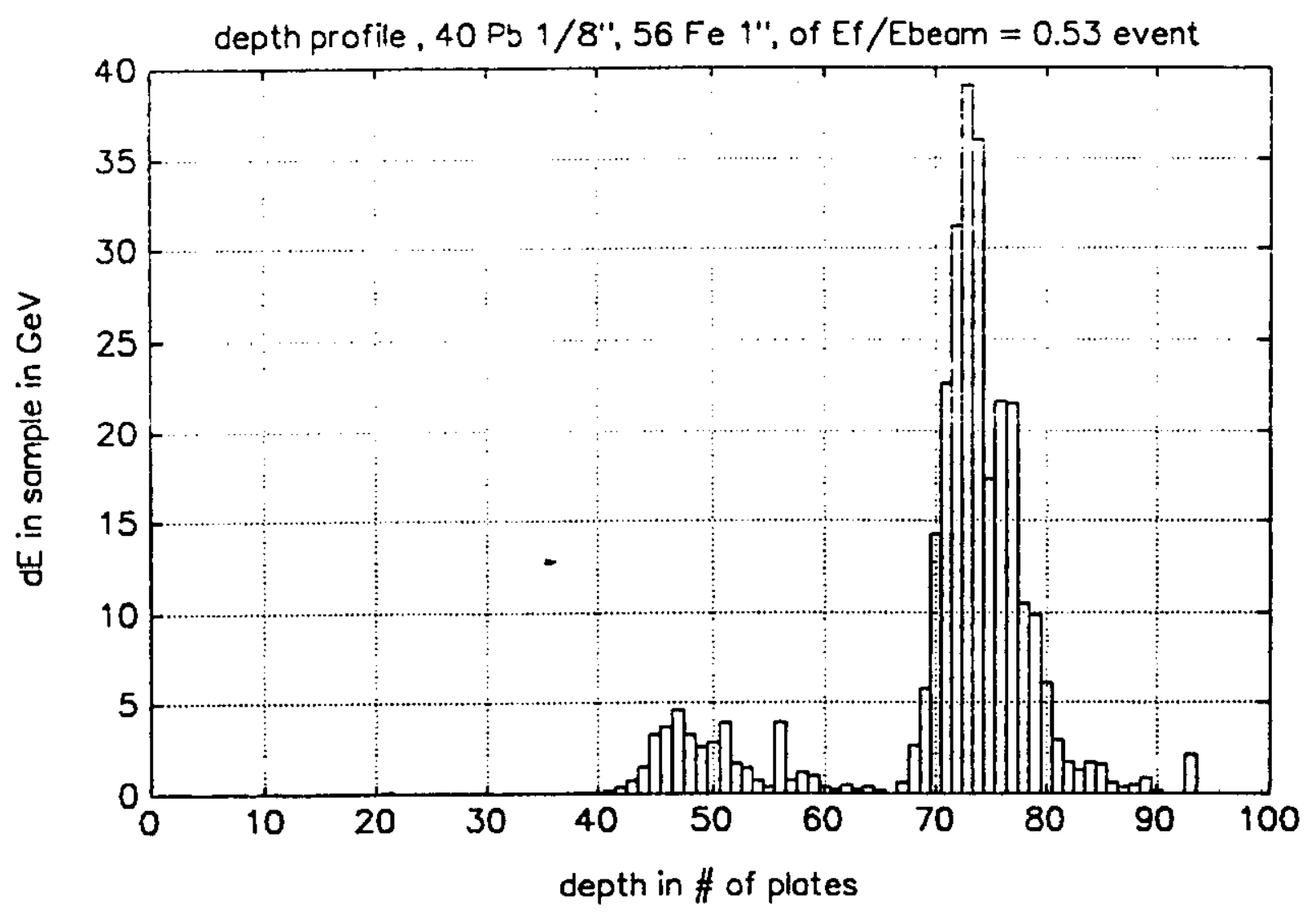

Figure 5. Profile of the badly fit $250 \mathrm{GeV}$ "hanging file" event. The EM compartment is 40 $\mathrm{Pb}$ plates $1 / 8^{\prime \prime}$ thick, while the hadronic compartment is 55 plates of 1 " Fe. Most of the energy is lost in the inert coil region. 


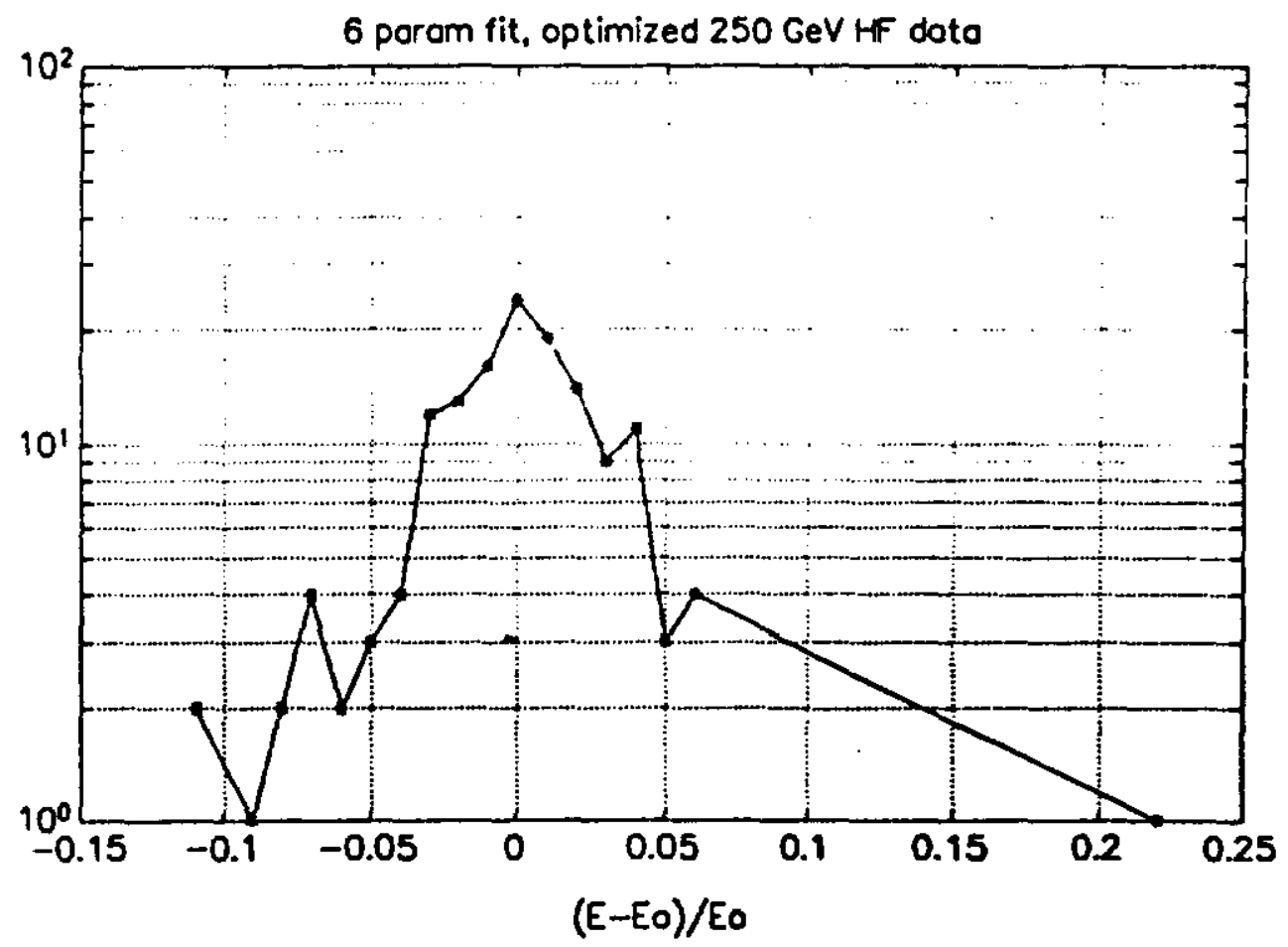

Figure 6. Fractional energy resolution for a 6 parameter fit to the measured energy in the $250 \mathrm{GeV}$ "hanging file" data. All events are plotted. 


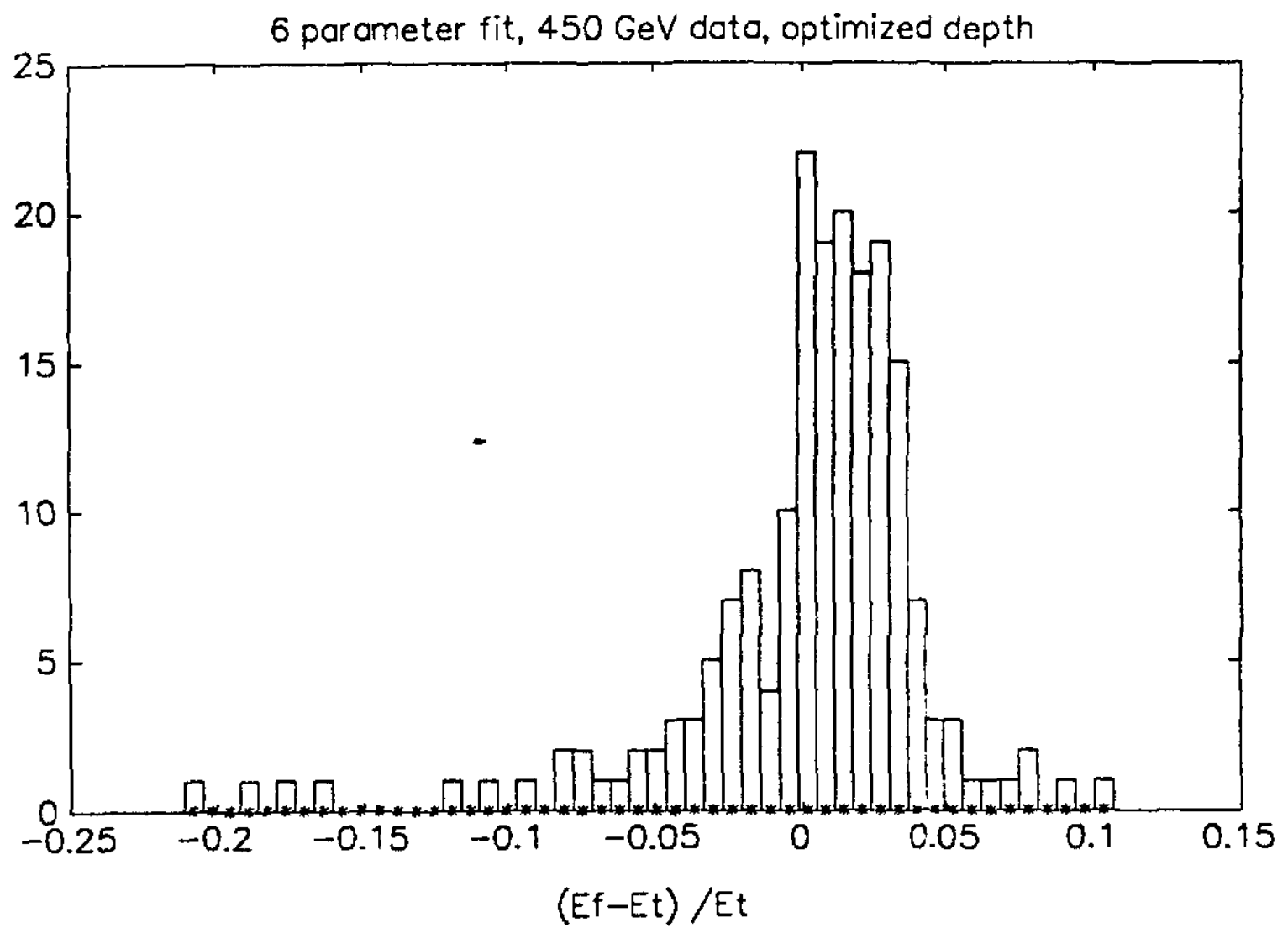

Figure 7. Fractional energy resolution for a 6 parameter fit to the measured energy in the $450 \mathrm{GeV}$ neutrino detector data. 

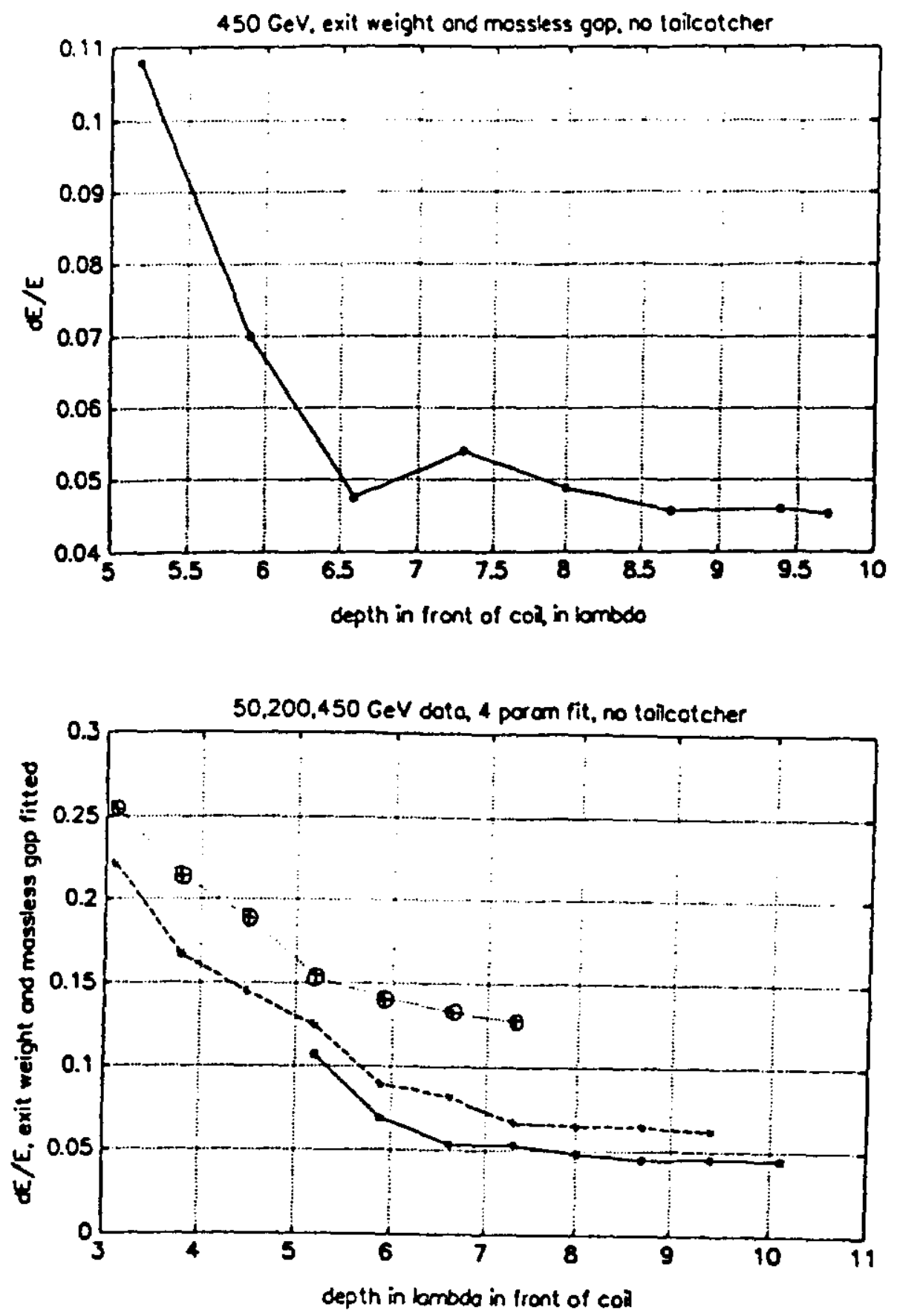

Figure 8. Fractional energy resolution for a 4 parameter fit to the measured energy in the neutrino detector data. The coil depth is 1.4 followed by a "massless gap". The depth 0.7 just upstream of the coil has independent "exit weight" readout. There is no tailcatcher absorber or readout.

a. $450 \mathrm{GeV}$ energy resolution as a function of depth upstream of the coil.

b. 50,200 , and $450 \mathrm{GeV}$ energy resolution as a function of the total depth upstream of the coil, plotted as points/line $\oplus \ldots, *_{-\ldots} \cdot-$ respectively. 